\title{
Gestational diabetes mellitus causes dyslipidemia in late trimester: mini review
}

\author{
Poonguzhalai S. ${ }^{1}$, Kalyanikutty K. P. ${ }^{2} *$
}

\author{
${ }^{1}$ Department of Physiology, JIPMER, Puducherry, India \\ ${ }^{2}$ Department of Physiology, Calicut Govt Medical College, Kerala, India
}

Received: 18 October 2019

Revised: 22 November 2019

Accepted: 28 November 2019

\author{
*Correspondence: \\ Dr. Kalyanikutty K. P., \\ E-mail: poongu85@gmail.com
}

Copyright: () the author(s), publisher and licensee Medip Academy. This is an open-access article distributed under the terms of the Creative Commons Attribution Non-Commercial License, which permits unrestricted non-commercial use, distribution, and reproduction in any medium, provided the original work is properly cited.

\begin{abstract}
The incidence of gestational diabetes mellitus (GDM) is increasing rapidly worldwide. Many women with gestational diabetes mellitus are likely to have type 2 diabetes. With the extensive management protocol for GDM we are able to obtain a good glycaemic control but still excess morbidity prevails among GDM pregnancy compared to normal pregnancy. This may be due to the dysfunction of lipid metabolism. Changes in carbohydrate and lipid metabolism occur during pregnancy to ensure a continuous supply of nutrients to the growing fetus despite intermittent maternal food intake. Exaggerated reduction in insulin sensitivity in the peripheral tissues combined with peripheral adipose tissue lipolysis in GDM pregnancy than normal pregnancy results in increased maternal lipoprotein concentrations and elevated lipoprotein triglyceride content. An altered lipid profile on the maternal side would modulate the quantity and quality of lipids being transferred to the fetus. Hypertriacylglycerolemia in gestational diabetes mellitus has been related to a significant risk of having neonates that are large for gestational age and it is considered as a major cause of preeclampsia in the late gestational age. So, the recent researchers emphasize on targeting lipid metabolism in pregnant women with GDM to avoid the adverse outcomes of pregnancy.
\end{abstract}

Keywords: Dyslipidaemia, Gestational diabetes mellitus, Large for date baby, Preeclampsia

\section{INTRODUCTION}

The incidence of gestational diabetes mellitus (GDM) is increasing rapidly worldwide. Many women with gestational diabetes mellitus are likely to have type 2 diabetes. We are able to obtain a good glycaemic control with the extensive management protocol for GDM but still excess morbidity prevails among GDM pregnancy compared to normal pregnancy. It has been acknowledged that elevated maternal lipids are associated with preeclampsia, preterm delivery, and large-for gestational-age infants in the pregnant women with GDM. ${ }^{1}$ The physiological changes in insulin and lipids are exaggerated in pregnancy with GDM and this may indicate the underlying metabolic dysfunction that transiently manifests during pregnancy. In this review we try to examine the association of dyslipidaemia with pregnancy complicated by GDM.

\section{REVIEW OF LITERATURE}

\section{Lipid and glucose metabolism in a pregnant women}

\section{Lipid metabolism in pregnant women}

Chylomicrons formed by the exogenous system of lipid metabolism are the products of fat digestion and this is absorbed by the intestinal mucosa. The chylomicrons are 
cleared from the circulation by the action of, action of lipoprotein lipase, which is located on the surface of the endothelium of the capillaries. The triglyceride in the chylomicrons are broken down to FFA (free fatty acid) and glycerol by the action of lipoprotein lipase. Chylomicrons depleted of their triglyceride remain in the circulation as cholesterol-rich lipoproteins called chylomicron remnants, which are carried to the liver, where they are internalized by receptor-mediated endocytosis by binding to chylomicron remnant and LDL receptors and are degraded in lysosomes. ${ }^{2}$

An endogenous system of lipid metabolism result in the formation of very low-density lipoproteins (VLDL), intermediate-density lipoproteins (IDL), low-density lipoproteins (LDL), and high-density lipoproteins (HDL), also transports triglycerides and cholesterol throughout the body. VLDL are formed in the liver and transport triglycerides formed from fatty acids and carbohydrates in the liver to extrahepatic tissues. After their triglyceride is largely removed by the action of lipoprotein lipase, they become IDL. Some IDL are taken up by the liver. The remaining IDL then lose more triglyceride and protein, probably in the sinusoids of the liver, and become LDL. LDL provide cholesterol to the tissues where they are taken up by receptor-mediated endocytosis. $^{2}$

In the steady state, cholesterol leaves as well as enters cells and this cholesterol is taken up by HDL. ${ }^{3}$ These lipoproteins are synthesized in the liver and the intestine. The HDL system transfers cholesterol to the liver, which is then excreted in the bile. In this way, it lowers plasma cholesterol. Thus, the free fatty acid (FFA) are provided to fat cells and other tissues by chylomicrons and VLDL. ${ }^{4}$ They are also synthesized in the fat depots in which they are stored. They circulate bound to albumin and are a major source of energy for many organs., ${ }^{2,3}$

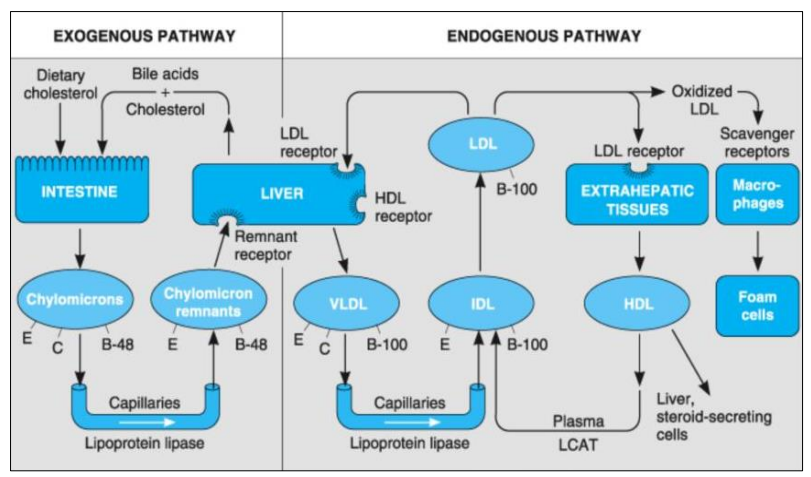

Figure 1: Exogenous and the endogenous pathway of lipid metabolism. ${ }^{3}$

The supply of FFA to the tissues is regulated by two lipases. As noted above, lipoprotein lipase on the surface of the endothelium of the capillaries hydrolyzes the triglycerides in chylomicrons and VLDL, providing FFA and glycerol, which are reassembled into new triglycerides in the fat cells. The intracellular hormonesensitive lipase of adipose tissue catalyzes the breakdown of stored triglycerides into glycerol and fatty acids, with the latter entering the circulation (Figure 1). ${ }^{3,4}$

\section{Adipose tissue metabolism and the placental transfer of lipids}

The body fat gets accumulated during the early pregnancy as long chain Poly unsaturated fatty acid (LC PUFA) from the maternal diet and maternal metabolism. In the late stage of pregnancy the lipolytic activity mediated by hormone sensitive lipase of adipose tissue increases releasing more fatty acid into the circulation which enters the liver for the synthesis of triglycerides or triacylglycerol (TG) so there is increased release of TG rich lipoproteins like VLDL into the circulation. ${ }^{5}$ This VLDL is not removed from the circulation due to reduced lipoprotein lipase. ${ }^{6}$ During early pregnancy, long-chain polyunsaturated fatty acids (LC-PUFA) may accumulate in maternal fat depots and become available for placental transfer during late pregnancy when the fetal growth rate is maximal and fetal requirements for LC-PUFAs are greatly enhanced. During this late part of gestation, enhanced lipolytic activity in adipose tissue contributes to the development of maternal hyperlipidemia, there is an increase in plasma triacylglycerol concentrations, with smaller rises in phospholipid and cholesterol concentrations. Besides the increase in plasma very-lowdensity lipoprotein, there is a proportional enrichment of triacylglycerol in both low-density lipoproteins and highdensity lipoproteins. These lipoproteins transport LCPUFA in the maternal circulation. ${ }^{6}$ The presence of lipoprotein receptors in the placenta allows their placental uptake, where they are hydrolysed by lipoprotein lipase, phospholipase A and intracellular lipase. The fatty acids that are released can be metabolized and diffuse into the fetal plasma. Although present in smaller proportions, maternal plasma non-esterified fatty acids are also a source of LC-PUFA for the fetus, their placental transfer being facilitated by the presence of a membrane fatty acid-binding protein. There is very little placental transfer of glycerol, whereas the transfer of ketone bodies may become quantitatively important under conditions of maternal hyperketonaemia, such as during fasting, a highfat diet or diabetes. ${ }^{5,7}$ The demands for cholesterol in the fetus are high, but maternal cholesterol substantially contributes to fetal cholesterol during early pregnancy but fetal cholesterol biosynthesis is rather main mechanism for satisfying fetal requirements during late trimester than cholesterol transfer from maternal lipoproteins. The LDL and HDL are enriched with TG by two other mechanisms that take place in the mid pregnancy. There is increased activity of cholesterol ester transfer protein (CETP) that mediates transfer of TG from VLDL to LDL and HDL lipoproteins. Conversion of TG rich HDL2b to HDL3 which is rich in esterified cholesterol is reduced due to decreased activity of hepatic lipase (HL). All these lead to increased plasma triacylglycerol in the maternal circulation during the last trimester (Figure 2). ${ }^{7,8}$ 


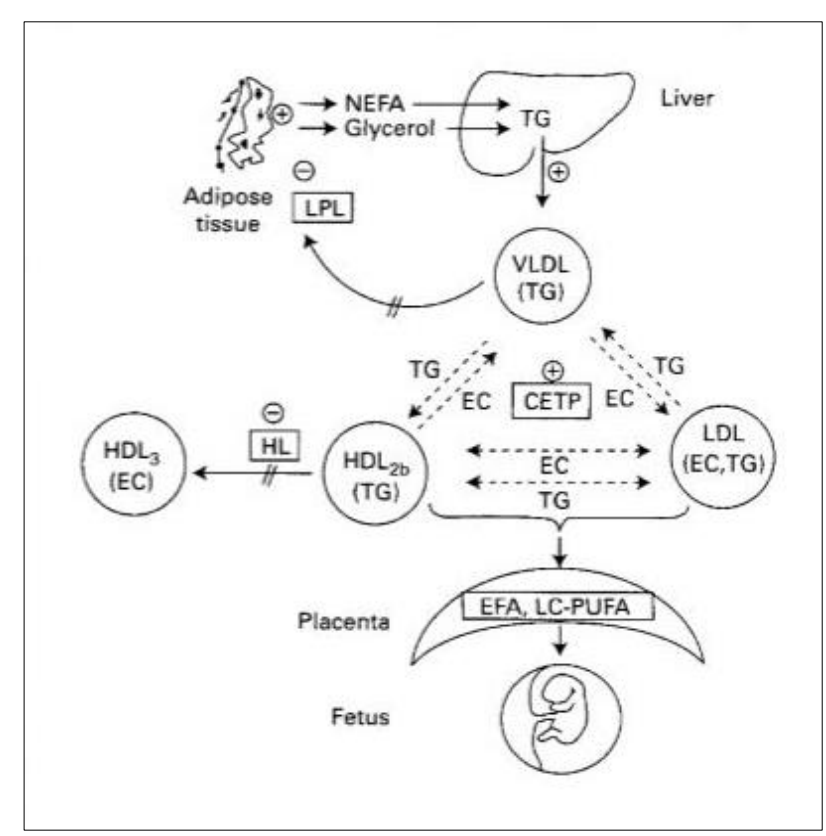

FFA - Free Fatty acid, FAT/CD36 - Fatty acid translocase, FATP - Fatty acid transport protein, FABPpm - Plasma membrane fatty acid binding protein, LR - Lipoprotein receptor, LRP - Lipoprotein related protein, SR-A - Scavenger receptor type A, SR B1 - Scavenger receptor class B type 1.

Figure 2: Proposed mechanism for increased triaclylglycerol in the maternal circulation during the last trimester. ${ }^{11}$

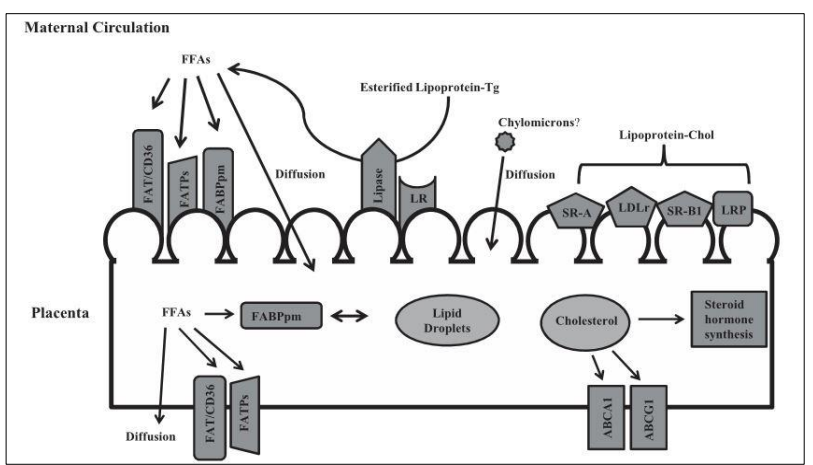

NEFA - Non esterified fatty acid, LPL - Lipoprotein lipase, TG - Triglycerides, VLDL - Very low-density lipoprotein, CETP Cholesterol ester transfer protein, HL - Hepatic lipase, HDL High density lipoprotein, EFA - Esterified fatty acid, EC Esterified cholesterol, LC PUFA - Long chain polyunsaturated fatty acid.

Figure 3: Proposed mechanism of placental transfer of lipids to the fetus. ${ }^{1,15}$

Thus, formed maternal free fatty acids (FFAs) can be directly taken up into the trophoblast by the action of lipoprotein lipases, that release FFA from the maternal circulating lipoproteins. The trophoblast expresses receptors for VLDL cholesterol (VLDL receptor), LDL-C (LDL receptor), and HDL-C [scavenger receptor class $B$ type 1 (SR-B1)] on the maternal side of synchiotrophoblast that mediates the uptake of lipoproteins along the placenta. The placenta takes up cholesterol from the maternal circulation. This cholesterol is used in steroid synthesis in the placenta and is transported to the fetus. ${ }^{8}$ The denova synthesis of cholesterol is predominant in the fetus than the placental transfer of cholesterol also takes up cholesterol. FFAs move to the fetal circulation by facilitated diffusion or using fatty acid translocase (FAT/CD36) and fatty acid transport protein (FATP) in the fetal side of syncytiotrophoblast basal membrane. Cholesterol is moved out of the trophoblast by secretion of lipoproteins, through complex formation with apoE, and efflux by SRB1, ABCA1, and ABCG1 which are expressed on the fetal side of the trophoblast. Direct uptake of chylomicron remnant particles has been demonstrated in mouse placenta, raising the possibility that this may also occur in human pregnancy (Figure 3). 8,9

\section{Glucose metabolism in pregnant women}

Glucose metabolism mainly aim at providing sufficient nutrients to the developing fetus. During early pregnancy, glucose tolerance is normal or slightly improved and peripheral insulin sensitivity and hepatic basal glucose production is normal. It is almost same as that of pregravid values. As gestational age progresses the fetoplacental unit develops and start secreting the placental hormones such as human chorionic somatomammotropin (HCS), progesterone, cortisol, and prolactin. This hormonal milieu brings about the insulin resistance state and impaired $\beta$ cell response to increased hepatic gluconeogenesis in the late gestational age. Prevailing insulin resistance produces exaggerated changes in postprandial concentrations of metabolic fuels (e.g., glucose, VLDL, and amino acids). ${ }^{3,10}$ Insulin resistance serves to shunt ingested nutrients to the fetus after feeding. In addition to maternally and placentally derived hormones, changes in the production of inflammatory mediators by the placenta (e.g., tumor necrosis factor-a) and cytokines produced by adipose tissue also contribute to the decrease in insulin sensitivity in peripheral tissues. The levels of the adipocyte-derived hormone leptin are found to be increased in the late gestational age also contribute to the insulin resistant state. ${ }^{11-13}$

\section{GDM cause dyslipidemia}

Changes in carbohydrate and lipid metabolism occur during pregnancy to ensure a continuous supply of nutrients to the growing fetus despite intermittent maternal food intake. These metabolic changes are progressive and may be accentuated in women who develop gestational diabetes mellitus (GDM). Exaggerated reduction in insulin sensitivity in the peripheral tissues combined with peripheral adipose tissue lipolysis in GDM pregnancy than normal pregnancy results in increased maternal lipoprotein concentrations and elevated lipoprotein triglyceride content, including VLDL, HDL, and LDL. ${ }^{12}$ There is concomitantly increased maternal hepatic 
gluconeogenesis and preferential maternal utilization of ketones in the fasting state, freeing maternal glucose for use as the primary substrate for fetal energy production. An altered lipid profile on the maternal side would modulate the quantity and quality of lipids being transferred to the fetus. Hypertriacylglycerolemia in gestational diabetes mellitus has been related to a significant risk of having neonates that are large for gestational age. ${ }^{13}$ The cord blood glucose, NEFA, insulin and HOMA were higher in GDM than in controls suggesting that maternal lipids are strong predictors of fetal lipids and fetal growth. This mechanism is also facilitated by the secretion of certain adipocytokines from the adipose tissue. ${ }^{14-16}$

Adipocyte fatty acid-binding protein (AFABP) is secreted from the adipose tissue. This protein is responsible for intracellular fatty acid trafficking. In pregnant women it has been found that there are higher concentrations of AFABP in GDM than in control subjects thus favouring the increased transfer of FFA to the fetus giving rise to increased fat mass in fetus. ${ }^{17}$ Another protein leptin is secreted from the adipose tissue and placenta. The plasma concentration of leptin is found to be more in GDM than control subject and is strongly correlated to fetal macrosomia. ${ }^{14}$ Retinol binding protein 4 (RBP4) is another adipocytokine secreted from adipose tissue and placenta. RBP4 is found to higher in GDM pregnant women not only correlate with macrosomia but also with the insulin resistant state in them. ${ }^{18}$ Adiponectin is another protein that is secreted from the adipocyte and many fetal tissue also correlate with macrosomia. ${ }^{19}$ Angiopoietin like protein 4 (ANGPTL4) is the protein that is secreted from adipose tissue, liver and placenta is found to inhibit the LPL activity in the placenta. Normally LPL has been associated with the transport of fatty acid to the fetus. There is an enhanced LPL activity in the placenta at late pregnancy in GDM and this process is facilitated by the reduction of ANGPTL4 in the maternal circulation. ${ }^{20}$ Inadequate placental supply of free fatty acid and dysfunction of placental endothelial lipase and LPL has been reported in IUGR pregnancies. All these findings are suggestive of the role of lipids in macrosomia in the pregnant women with GDM. ${ }^{21}$

GDM also increases the risk of preeclampsia in the late gestational period. Increase in triacylglycerol during the last trimester is the underlying cause for insulin resistance and endothelial dysfunction which may lead to the development of preeclampsia. ${ }^{22}$ Though many studies suggest the increasing prevalence of preeclampsia in pregnant women with GDM, further research is needed in examining the predictive markers, pathophysiology, treatment and the long term health implications of preeclampsia in this population. ${ }^{23}$

\section{DISCUSSION}

There is an evidence that abnormal maternal lipids in pregnant women with GDM are associated with adverse pregnancy outcomes for mother and infant. ${ }^{18}$ Treating GDM to have a good glycemic control is mandatory till date but recent researchers emphasize on targeting lipid metabolism in pregnant women with GDM to avoid the adverse outcomes of pregnancy. ${ }^{1}$ Therefore considerable work has to be done in assessing the pathophysiology underlying dyslipidaemia in GDM before we can routinely adjust maternal lipids in pregnancy.

Funding: No funding sources Conflict of interest: None declared

Ethical approval: Not required

\section{REFERENCES}

1. Barrett HL, Dekker Nitert M, McIntyre HD, Callaway LK. Normalizing metabolism in diabetic pregnancy: is it time to target lipids? Diab Care. 2014;37(5):1484-93.

2. Herrera E, Ortega-Senovilla H. Lipid metabolism during pregnancy and its implications for fetal growth. Curr Pharm Biotechnol. 2014;15(1):24-31.

3. Ganong WF. Review of medical physiology. $22^{\text {nd }} \mathrm{Mc}$ Graw Hill; 2005:308-311.

4. Murray RK, editor. Harper's illustrated biochemistry. $26^{\text {th }}$ editions. New York, NY: (A Lange medical book). Lange Medical Books/McGraw-Hill; 2003:219-230.

5. Williams RH, Kronenberg $\mathrm{H}$, editors. Williams textbook of endocrinology: website included. $11^{\text {th }}$ edition, Philadelphia, Pa: Saunders, Elsevier; 2008:1589.

6. Butte NF. Carbohydrate and lipid metabolism in pregnancy: normal compared with gestational diabetes mellitus. The Am J Clin Nutrit. 2000;71(5):1256-61.

7. Ryckman KK, Spracklen CN, Smith CJ, Robinson JG, Saftlas AF. Maternal lipid levels during pregnancy and gestational diabetes: a systematic review and meta-analysis. Int $\mathrm{J}$ Obstet Gynaecol. 2015;122(5):643-51.

8. Wulf P. Maternal-fetal cholesterol transport in the placenta. Circ Res. 2009;104(5):569-71.

9. Gil-Sánchez A, Demmelmair H, Parrilla JJ, Koletzko B, Larqué E. Mechanisms involved in the selective transfer of long chain polyunsaturated fatty acids to the fetus. Front Genet. 2011;2:57.

10. Madsen EM, Lindegaard MLS, Andersen CB, Damm $P$, Nielsen LB. Human placenta secretes apolipoprotein B-100-containing lipoproteins. J Biol Chem. 2004;279(53):55271-6.

11. Herrera E, Amusquivar E, López-Soldado I, Ortega H. Maternal lipid metabolism and placental lipid transfer. Horm Res. 2006;65 Suppl 3:59-64.

12. Desoye G, Gauster M, Wadsack C. Placental transport in pregnancy pathologies. Am J Clin Nutr. 2011;94(6 Suppl):1896S-1902S.

13. Angueira AR, Ludvik AE, Reddy TE, Wicksteed B, Lowe WL, Layden BT. New insights into gestational 
glucose metabolism: lessons learned from $21^{\text {st }}$ century approaches. Diabetes. 2015;64(2):327-34.

14. Okereke NC, Uvena-Celebrezze J, Hutson-Presley L, Amini SB, Catalano PM. The effect of gender and gestational diabetes mellitus on cord leptin concentration. Am J Obstet Gynecol. 2002;187(3):798-803

15. Herrera E, Ortega-Senovilla H. Disturbances in lipid metabolism in diabetic pregnancy - are these the cause of the problem? Best Pract Res Clin Endocrinol Metab. 2010;24(4):515-25.

16. Rebholz SL, Burke KT, Yang Q, Tso P, Woollett LA. Dietary fat impacts fetal growth and metabolism: uptake of chylomicron remnant core lipids by the placenta. Am J Physiol Endocrinol Metab. 2011;301(2):E416-425.

17. Xu A, Wang Y, Xu JY, Stejskal D, Tam S, Zhang J, et al. Adipocyte fatty acid-binding protein is a plasma biomarker closely associated with obesity and metabolic syndrome. Clin Chem. 2006;52(3):405-13.

18. Choi SH, Kwak SH, Youn BS, Lim S, Park YJ, Lee $\mathrm{H}$, et al. High plasma retinol binding protein-4 and low plasma adiponectin concentrations are associated with severity of glucose intolerance in women with previous gestational diabetes mellitus. J Clin Endocrinol Metab. 2008;93(8):3142-8.
19. Kadowaki K, Waguri M, Nakanishi I, Miyashita Y, Nakayama M, Suehara N, et al. Adiponectin concentration in umbilical cord serum is positively associated with the weight ratio of fetus to placenta. J Clin Endocrinol Metab. 2006;91(12):5090-4.

20. Kersten S. Regulation of lipid metabolism via angiopoietin-like proteins. Biochem Soc Trans. 2005;33(Pt 5):1059-62.

21. Bharathi KR, Vijayalakshmi S, Shrunga RP. A study of lipid parameters among GDM and non GDM pregnant women: a hospital-based study. Int J Reprod Contracept Obstet Gynecol. 2017;6:5488-90.

22. Weissgerber TL, Mudd LM. Preeclampsia and Diabetes. Curr Diab Rep. 2015;15(3):579.

23. Sullivan SD, Umans JG, Ratner R. Hypertension complicating diabetic pregnancies: pathophysiology, management, and controversies. J Clin Hyper (Greenwich). 2011;13(4):275-84.

Cite this article as: Poonguzhalai S, Kalyanikutty

KP. Gestational diabetes mellitus causes

dyslipidemia in late trimester: mini review. Int J

Reprod Contracept Obstet Gynecol 2020;9:453-7. 